\title{
A REAL-TIME PROTEIN DETECTOR UTILIZING THE VROMAN EFFECT ON SAM-FUNCTIONALIED SURFACES
}

\author{
Seokheun Choi, Yongmo Yang, and Junseok Chae \\ Electrical Engineering, Arizona State University, Tempe, AZ, USA
}

\begin{abstract}
We report a new SPR (surface plasmon resonance) protein sensor using the Vroman effect for real-time, sensitive and selective detection of protein. The sensor relies on the competitive nature of protein adsorption to the surface, directly depending upon protein's molecular weight. We, for the first time, utilize the Vroman effect for real-time protein microsensors using three different proteins including annexin (36 kDa), streptavidine (53 kDa), and isolectin (114 kDa) on three different surfaces which are a bare-gold surface and two others modified by $\mathrm{OH}$ - and $\mathrm{COOH}$-terminated SAM (Self Assembly Monolayer). The real-time adsorption and displacement of the proteins are monitored by SPR demonstrating very high sensitivity and selectivity label-free protein sensor. The biosensor can distinguish at least $17 \mathrm{kDa}$ in molecular weight with $100 \%$ selectivity. The protein detector can be integrated with microfluidic systems to provide extremely sensitive and selective analytical capability.
\end{abstract}

\section{INTRODUCTION}

Biosensors comprise a specific bio-receptor and a sensitive transducer. The bio-receptor requires high selectivity and affinity whereas the transducer demands high sensitivity and large dynamic range. Depending upon detection mechanisms, biosensors can largely be categorized as either labeled or label-free system. Labeling techniques have been broadly employed to allow automation of detection process. However, the chemical labeling may modify the target proteins characteristics so that their natural activity is impaired [1]. Moreover, the labeling is time-consuming and labor-intensive and often it is difficult to achieve accurate quantification due to different labeling efficiency for different proteins. Label-free system, on the other hand, does not require labeling process and has started to be attractive for biological analysis [2]. Among various label-free mechanisms, SPR has been one of the leading techniques due to its extremely high sensitivity (The detection limit of the SPR is up to a few ppt (pg mL $\left.{ }^{-1}\right)$ ) [3]. For selectivity, researchers use antibodies, protein lysates, lectins, peptides, and aptamers, which are single stranded DNAs or RNAs synthesized in a combinatorial fashion, as bio-receptors [4]. However, weak binding affinity with analytes, non-specific adsorption, and low reproducibility still remains critical limitations.

In this paper, we present a new proof-of-concept high sensitivity and selectivity SPR protein sensor operating without using a bio-receptor. The selectivity, typically offered by bio-receptors, is provided by a competitive nature of protein adsorption to the surface, directly depending upon protein's molecular weight, namely Vroman effect [5]. Leo Vroman first observed that fibrinogen adsorbed from plasma disappeared within minutes of surface contact. The loss was the first described example of the fibrinogen displacement phenomenon by other high molecular weight plasma proteins. Fig. 1 describes the Vroman effect; a low-molecular-weight (LMW) protein, initially covers the surface, is displaced by a high-molecular-weight (HMW) protein. It is more energetically stable when HMW proteins replace LMW ones in nature. However, when HMW proteins cover the surface first, LMW ones arriving later do not displace the former. We utilize the molecular weight differences as selectivity of our biosensor.

In section 2, surface preparation and device fabrication are discussed. Experimental and evaluation methods are presented in section 3. In section 4, we demonstrate SPR measurements results using three different proteins. Finally, concluding remarks follow in section 5 .

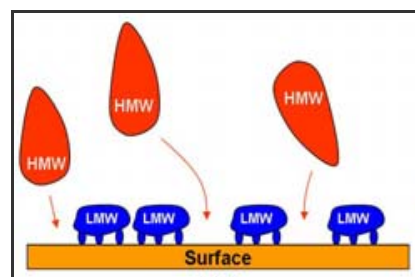

(a)

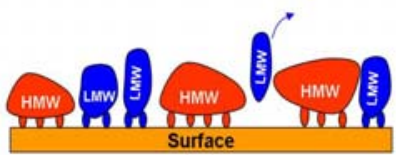

(c)

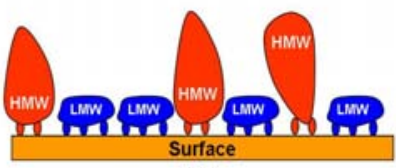

(b)

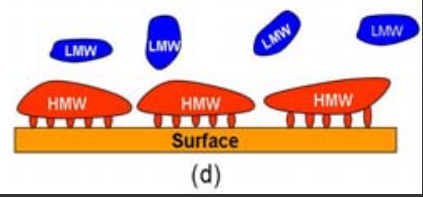

(d)
Figure 1. Schematic of the Vroman effect

\section{SURFACE PREPARATION AND MICROFLUIDIC DEVICE FABRICATION}

We use three different proteins; annexin (36 kDa), streptavidine (53 kDa), and isolectin $(114 \mathrm{kDa})$. All proteins are commercially available from Sigma Aldrich and Invitrogen. The proteins are diluted using Phosphate Buffered Saline (PBS) 1X (Mediatech Inc.).

We prepare three different surfaces; bare-gold, OH-terminated $\mathrm{SAM}$, and $\mathrm{COOH}$-terminated SAM. This is to observe Vroman effect on three different hydrophobicity surfaces. Bare-gold is the most hydrophobic and OH-SAM provides the most hydrophilic surfaces. Vroman effect occurs based upon energy preference of hydrophobicity / hydrophilicity surfaces; thus the surface modification greatly affects biosensor performance. Details are presented in section 4. Glass substrates $(B K 7, n=1.517)$ are first cleaned in piranha solution (a 3:1 ratio of $\mathrm{H}_{2} \mathrm{SO}_{4}$ and $\mathrm{H}_{2} \mathrm{O}_{2}$ ) for 10 min. The cleaned glass is coated with $\mathrm{Cr} / \mathrm{Au}(3 \mathrm{~nm} / 48 \mathrm{~nm})$ by thermal evaporation. Then the substrates are cleaned by oxygen plasma (Harrick Plasma Inc.) and immersed in an ethanol solution of different alkane-thiols at $1 \mathrm{mM}$ for $24 \mathrm{~h}$ at room temperature to form SAMs. COOH- and $\mathrm{OH}$ - terminated SAMs are formed on the substrate with 11-mercaptoundecanoic acid and 11-mercapto-1-undecanol. Finally the substrates are rinsed with ethanol and water, and thoroughly dried using nitrogen.

\subsection{Surface Characterization}

The three different surfaces are characterized by contact angle measurement and FTIR (Fourier Transform Infrared Spectroscopy). A droplet (2ul) of water is dispensed on the surfaces and the contact angle is measured (Easy Drop FM 40, Krüss GmbH Inc.). The contact angles for bare-gold, $\mathrm{COOH}$-, and $\mathrm{OH}$-terminated SAM are $82.6 \pm 0.77^{\circ}, 41.4 \pm 0.63^{\circ}$, and $38.2 \pm 0.32^{\circ}$, respectively. As expected, the bare-gold surface is very hydrophobic and $\mathrm{OH}$ - and $\mathrm{COOH}-\mathrm{SAM}$ surfaces are hydrophilic. The contact angle difference 
between $\mathrm{OH}$ - and $\mathrm{COOH}$ - SAM surfaces is not large; yet this small difference makes significant impact on the protein adsorption / desorption.

The compositions of the SAM surfaces are determined by FTIR (Nicolet Continuum, Thermo Electron Corp.). Figure 2 shows that the methylene stretching of the alkyl chains for both $\mathrm{COOH}-$ and $\mathrm{OH}$ - terminal SAMs are clearly visible with two absorption bands at 2917 and $2846 \mathrm{~cm}^{-1}$. The spectrum of the COOH-SAM shows the $\mathrm{C}=\mathrm{O}$ stretching band at $1706 \mathrm{~cm}^{-1}$ in the mid-IR spectrum, which suggest most terminal acid groups participate in intermolecular hydrogen bonding process. The OH-SAM spectrum shows the C-O stretch absorption at $1040 \mathrm{~cm}^{-1}$, thus validating the formation of SAMs on gold substrate.

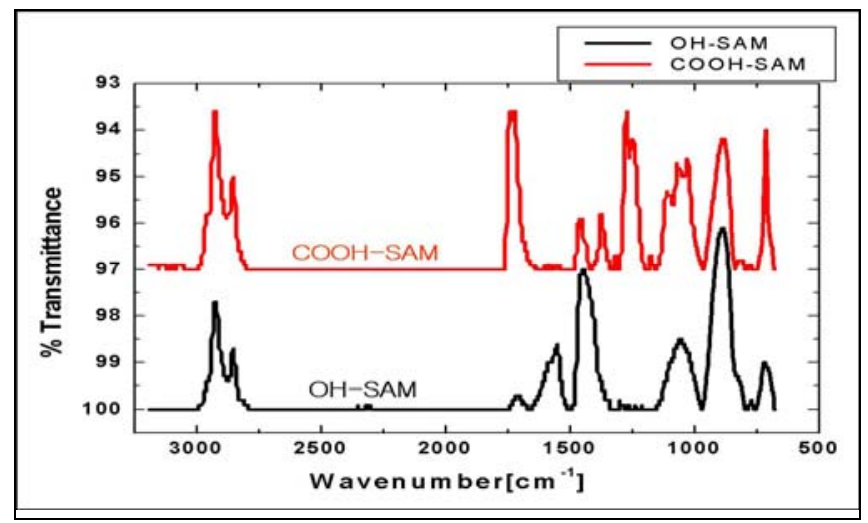

Figure 2. FTIR spectra of $\mathrm{OH}$ - and $\mathrm{COOH}-\mathrm{SAMs}$

\subsection{Microfluidic Device}

In order to facilitate potential integration with microfluidic systems, the sensor is enclosed by microfluidic channels/chambers (Figure. 3). On a Pyrex glass substrate (500um), $\mathrm{Cr} / \mathrm{Au}$ are deposited (3nm/48nm). A cover glass is patterned and etched to have $1 \mathrm{~mm}$ wide and 50um deep fluidic channels. Ports for inlets and outlets are $9.7 \mathrm{~mm}$ tall and $6.4 \mathrm{~mm}$ in diameter. The Pyrex substrate and cover glass are bonded by epoxy thin films (Upchurch Scientific) and cured at $120^{\circ} \mathrm{C}$ for $20 \mathrm{~min}$.

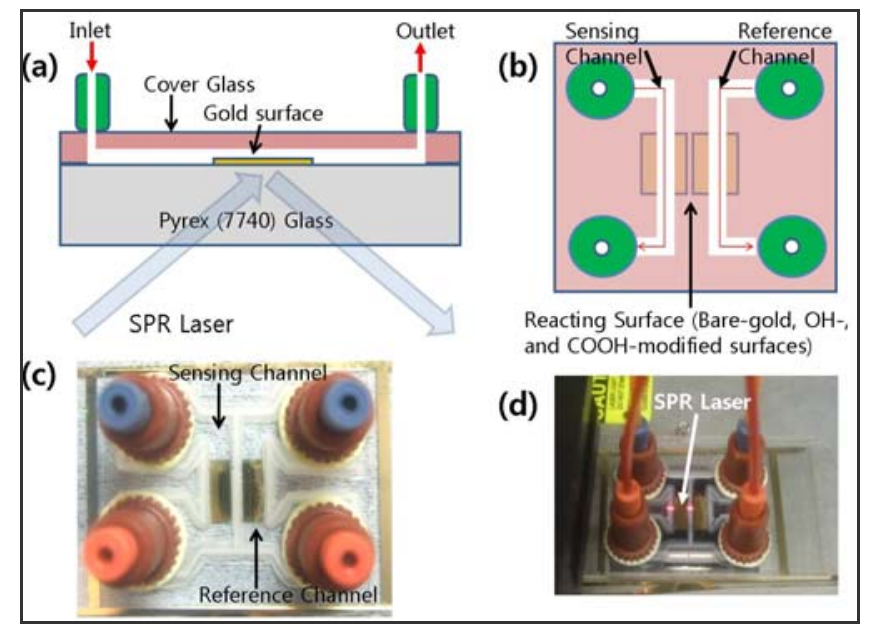

Figure 3. Schematics of (a) the cross sectional view, (b) the top view, (c) microfluidic enclosure, and (d) the bio-sensor chip on a SPR

\section{EXPERIMENTAL AND EVALUATION METHODS}

The fabricated device is mounted on SPR analytical system (Biosensing Instrument Inc.). We monitor the angle shift in real time as protein solution flows through the channels driven by an external syringe pump. First, PBS is circulated for 20 min until the SPR output stabilizes. The protein solution (1uM) flows through the channels at $20 \mathrm{ml} / \mathrm{min}$, which generates the angle shift proportional to molecular interactions on the surface. When protein absorption completes, we let PBS wash the surface to remove excess weakly bound proteins. As shown in figure 4, in order to observe Vroman effect, we first flow LMW proteins such as annexin $(36 \mathrm{kDa})$ or streptavidine $(53 \mathrm{kDa})$ to cover the surface. The bond strength depends upon the hydrophobicity of the surface; the more hydrophobic the surface is, the stronger the bond is [6]. Then, HMW proteins such as streptavidine $(53 \mathrm{kDa})$ or isolectin $(114 \mathrm{kDa})$ flow through the channels and replace existing bonds formed by the LMW proteins. We also perform the reverse configuration, HMW to LMW proteins, to confirm Vroman effect (figure 4 (3)).

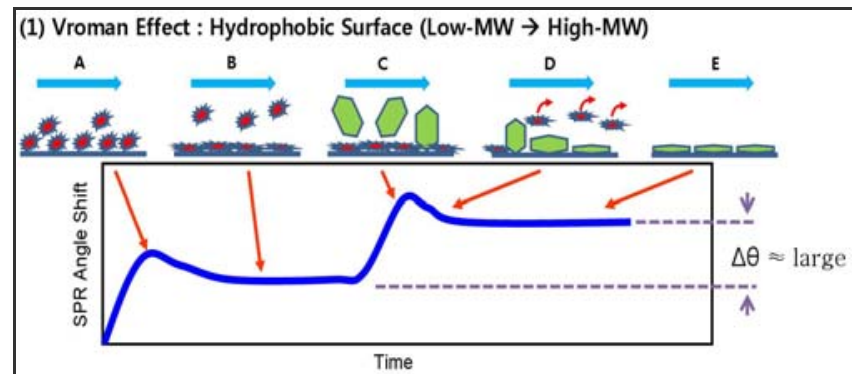

(2) Vroman Effect: Hydrophilic Surface (Low-MW $\rightarrow$ High-MW)
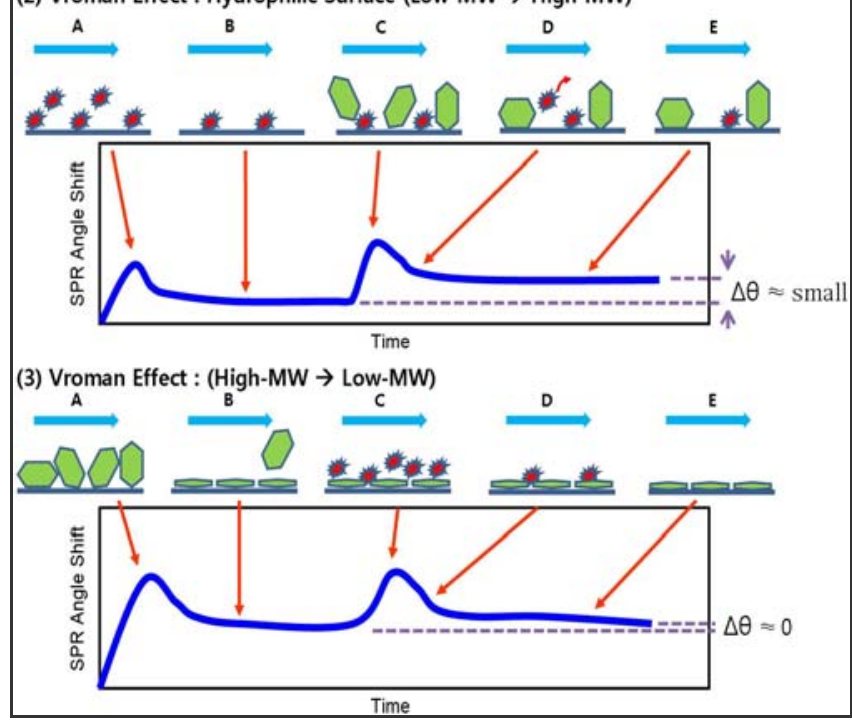

Figure 4. A schematic of SPR profiles \& illustration of the Vroman effect (1) on the hydrophobic and (2) the hydrophilic surfaces when the LMW protein adsorbs first and then the HMW protein arrives later to the surface. If the HMW protein adsorbs first and the LMW protein comes later, (3) no angle change occurs.

\section{RESULTS AND DISCUSSION}

The SPR angle shifts of each protein on three different surfaces (bare-gold, $\mathrm{COOH}$ - and $\mathrm{OH}$-terminated SAM) are listed in Table 1. The SPR angle shifts are proportional to the size of the proteins. HMW proteins generate larger angle shifts than LMW ones. 
Obviously, the larger proteins have stronger molecular interactions on the surfaces. Proteins that we use tend to adsorb more on the hydrophobic than hydrophilic surfaces. This phenomenon is due to the nature of proteins. Generally, proteins have hydrophobic residues buried within the core and their hydrophilic residues facing outside [7]. For any protein structure, when the protein adsorbs to a solid surface, the adsorption behavior is highly related to hydrophobic attraction [8]. Hydrophilic residues influence the orientation of the adsorbed protein, not absorption process. Therefore, the protein rapidly adsorbs to a hydrophobic surface, unfolds and spreads its hydrophobic residues over the surface. On the other hand, hydrophilic surfaces have weaker protein adsorption; thus, the adsorbed proteins are easily detached by subsequent PBS solution.

Table 1. The SPR angle shifts of proteins adsorption

\begin{tabular}{|c|c|c|c|}
\hline & \multicolumn{3}{|c|}{ SPR Angle Shift [mDeg] } \\
\cline { 2 - 4 } & Bare-gold & COOH-SAM & OH-SAM \\
\hline Annexin (36 kDa) & $195 \mathrm{mDeg}$ & $22 \mathrm{mDeg}$ & $8 \mathrm{mDeg}$ \\
\hline Streptavidine (53 kDa) & $380 \mathrm{mDeg}$ & $35 \mathrm{mDeg}$ & $11 \mathrm{mDeg}$ \\
\hline Isolectin (114 kDa) & $550 \mathrm{mDeg}$ & $72 \mathrm{mDeg}$ & $18 \mathrm{mDeg}$ \\
\hline
\end{tabular}

In order to monitor Vroman effect, it is important to saturate the surfaces. This is because HMW proteins adsorb the space between LMW proteins if the LMW proteins do not form a fully packed protein monolayer. We saturate the surfaces using $1 \mathrm{uM}$ proteins, more than enough to form a full packed monolayer. Figure 5 shows the SPR adsorption / displacement profiles of sequentially injected two proteins with different molecular weights on bare-gold, $\mathrm{COOH}-$ and $\mathrm{OH}-\mathrm{SAM}$, respectively. The angle shift increases at each protein adsorption and the peak decreases to stabilized angle after washing any weakly bound proteins from the surface. The final SPR angle shift represents fully adsorbed monolayer on the surfaces, the equilibrium state.

The monolayer of LMW proteins is displaced by subsequent HMW proteins, which produce the SPR angle shift. The angle shift demonstrates Vroman effect, indicating how many LMW proteins are replaced by HMW ones. Figure 6 presents the angle shifts of the three different proteins on the three different surfaces. Table 2 summarizes all experiments matrix. The larger the difference of molecular weight between LMW and HMW proteins is, the larger the angle shift is. Regardless of surface properties, we get the largest the angle shift from the smallest, annexin, to the largest protein, isolectin.

The angle shift also depends upon the surfaces; the hydrophilic surfaces have higher replacement percentage than the hydrophobic surface. On the bare-gold surface, LMW proteins strongly bind to the surface and be induced to conformational spreading. Subsequent HMW proteins do not replace all the adsorbed proteins from the suface. However, hydrophilic surfaces show much higher displacement ratio because weak bonds between the hydrophilic residues of the proteins and the surface do not induce the conformational spreading. The week bond allows the high percentage displacement.

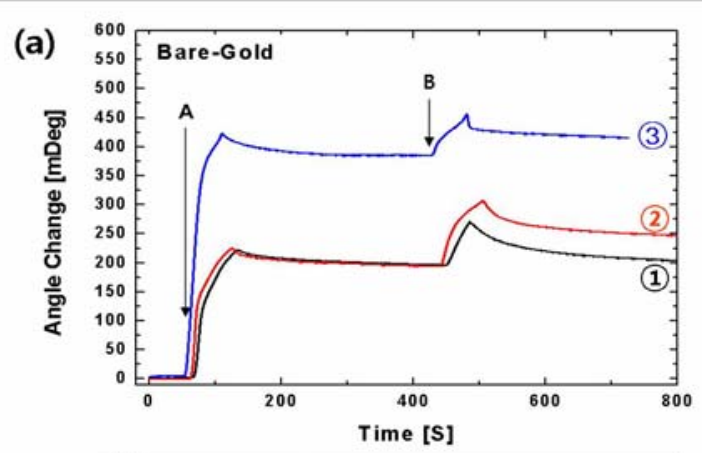

(b)

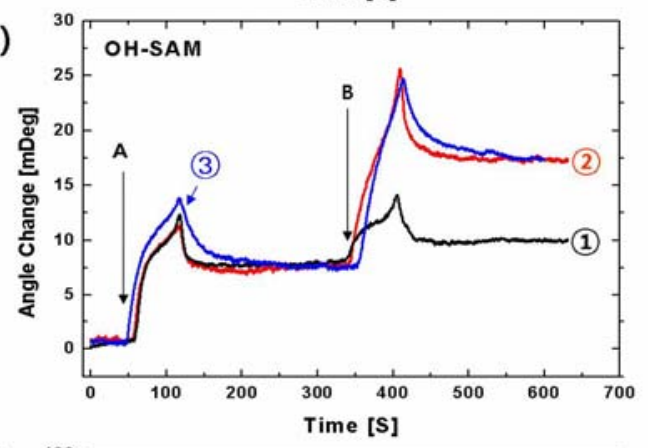

(c)
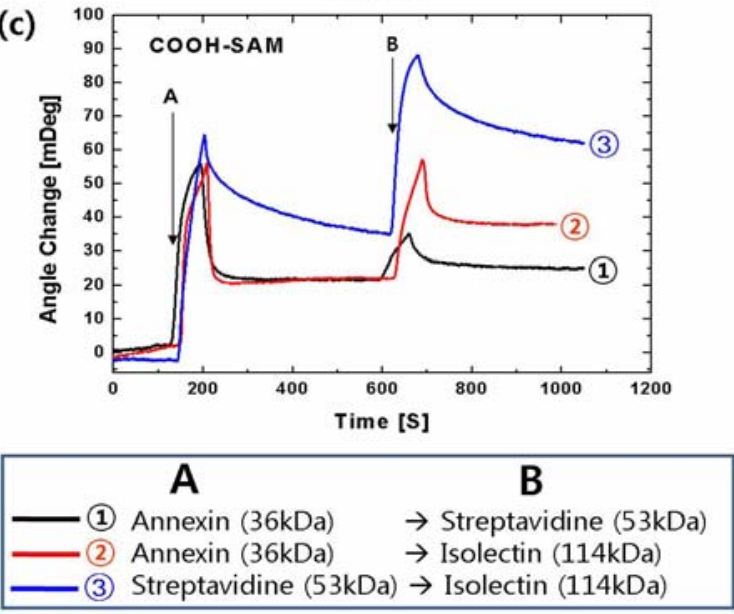

Figure 5. Real-time SPR adsorption profiles of proteins on the bare-gold, $\mathrm{OH}$ - and $\mathrm{COOH}-\mathrm{SAM}$ surfaces, respectively.

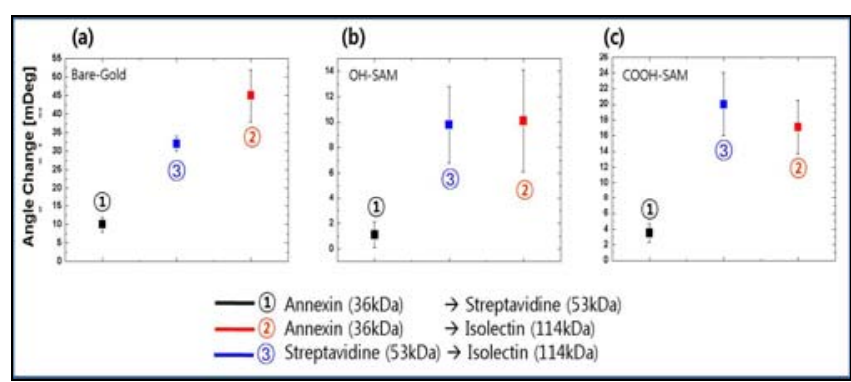

Figure 6. The SPR angle shifts on the bare-gold, $\mathrm{OH}$ - and $\mathrm{COOH}$-SAM surfaces, respectively. 
Table 2. Summary of the SPR angle shifts on the bare-gold, $\mathrm{OH}-$, and COOH-SAM surfaces. Angle shifts represent sensitivity and the sequential angle shifts indicates selectivity. Bare-gold shows the highest sensitivity yet limited selectivity. On the other hand $\mathrm{OH}$-SAM offers the best selectivity yet low sensitivity.

\begin{tabular}{|c|c|c|c|c|c|c|c|}
\hline & & \multicolumn{2}{|c|}{ Annexin $\rightarrow$ Isolectin } & \multicolumn{2}{|c|}{ Streptavidine $\rightarrow$ Isolectin } & \multicolumn{2}{|c|}{ Annexin $\rightarrow$ Streptavidine } \\
\hline & & $\begin{array}{l}\text { Annexin } \\
(36 \mathrm{kDa})\end{array}$ & $\begin{array}{l}\text { Isolectin } \\
(114 k D a)\end{array}$ & $\begin{array}{l}\text { Streptavidine } \\
(53 \mathrm{kDa})\end{array}$ & $\begin{array}{l}\text { Isolectin } \\
(114 \mathrm{kDa})\end{array}$ & $\begin{array}{l}\text { Annexin } \\
(36 \mathrm{kDa})\end{array}$ & $\begin{array}{l}\text { Streptavidine } \\
\quad(53 \mathrm{kDa})\end{array}$ \\
\hline \multirow{3}{*}{$\begin{array}{l}\text { Angle change } \\
\text { [mDeg] } \\
\& \\
\text { Rate of protein } \\
\text { displacement }\end{array}$} & $\begin{array}{l}\text { Bare-Gold } \\
\text { Substrate }\end{array}$ & $\begin{array}{c}++++ \\
(\sim 195 \mathrm{mDeg})\end{array}$ & $\begin{array}{c}++ \\
(\sim 50 \mathrm{mDeg}) \\
\sim 44 \% \text { displacement }\end{array}$ & $\begin{array}{c}+++++ \\
(380 \mathrm{mDeg})\end{array}$ & $\begin{array}{c}++ \\
(\sim 33 \mathrm{mDeg}) \\
\sim 75 \% \text { displacement }\end{array}$ & $\begin{array}{l}++++ \\
(-195 \\
\mathrm{mDeg})\end{array}$ & $\begin{array}{c}+ \\
(\sim 10 \mathrm{mDeg}) \\
\sim 54 \% \text { displacement }\end{array}$ \\
\hline & $\begin{array}{l}\mathrm{OH}-\mathrm{SAM} \\
\text { Substrate }\end{array}$ & $\stackrel{+}{+} \stackrel{+}{(\sim 8 \mathrm{mDeg}})$ & $\begin{array}{c}+ \\
(\sim 9 \mathrm{mDeg}) \\
-94 \% \text { displacement }\end{array}$ & $\stackrel{+}{+}$ & 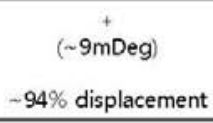 & $\stackrel{+}{+}$ & $\begin{array}{c}+ \\
(\sim 2 \mathrm{mDeg}) \\
\sim 91 \% \text { displacement }\end{array}$ \\
\hline & $\begin{array}{l}\text { COOH-SAM } \\
\text { Substrate }\end{array}$ & $\begin{array}{c}++ \\
(\sim 22 \mathrm{mDeg})\end{array}$ & $\begin{array}{c}++ \\
(\sim 16 \mathrm{mDeg}) \\
\sim 53 \% \text { displacement }\end{array}$ & $\begin{array}{c}++ \\
(\sim 35 \mathrm{mDeg})\end{array}$ & $\begin{array}{c}+++ \\
(\sim 27 \mathrm{mDeg}) \\
\sim 86 \% \text { displacement }\end{array}$ & $\begin{array}{c}++ \\
(\sim 22 \mathrm{mDeg})\end{array}$ & $\begin{array}{c}\stackrel{+}{(\sim 3 \mathrm{mDeg})} \\
\sim 71 \% \text { displacement }\end{array}$ \\
\hline
\end{tabular}

Angle change $[\mathrm{mDeg}]:+(0 \sim 10),++(11 \sim 50),+++(51 \sim 100)$,

$++++(101 \sim 200),+++++(>201)$

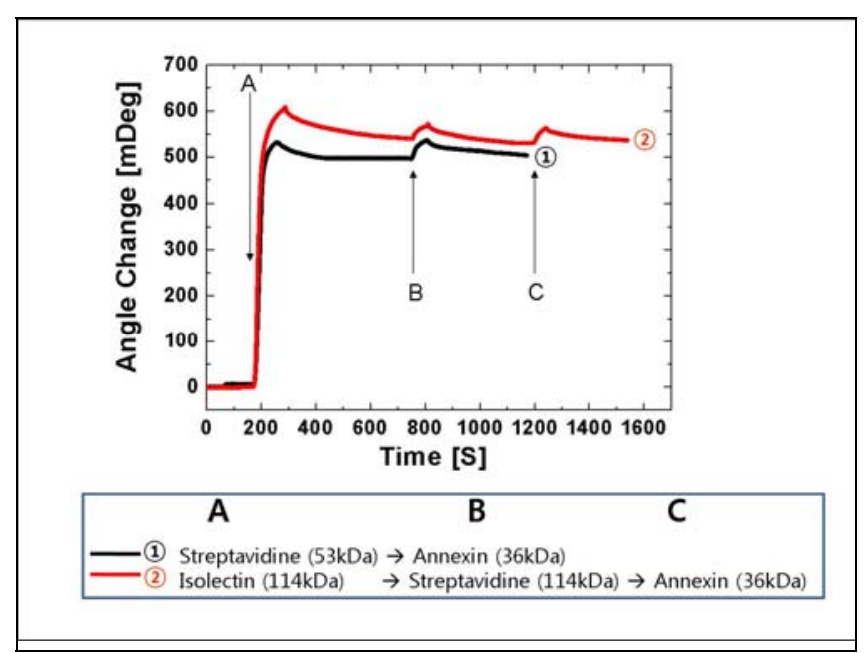

Figure 7. The SPR profiles of the opposite sequence injection (HMW proteins $\rightarrow$ LMW proteins)

In order to evaluate the selectivity of the protein sensor, we now reverse the proteins injection sequence; HMW proteins are injected first and then LMW ones arrive later. On all surfaces, the angle shifts are almost negligible, less than $0.3 \mathrm{mDeg}$, representing Vroman effect has nearly $100 \%$ selectivity as protein sensors. This data also support the SPR angle changes in figure 5 are not due to multilayer protein-protein interaction but solely due to Vroman effect.

\section{CONCLUSION}

Conventional protein sensors are limited to satisfy both high selectivity and sensitivity requirements at very low concentration because of complex structure and multiple forms of proteins. Existing protein sensors utilize a bio-receptor to capture target molecules; yet many challenges exist on integrating bio-receptors into the transducer. In this paper, we present a biosensor using Vroman effect. The biosensor utilizes a competitive nature of proteins adsorptions on the surfaces and read real-time molecular interactions using SPR. LMW proteins are displaced by HMW proteins, but the reverse sequence does not occur. Vroman effect and SPR offer extremely high selectivity and sensitivity. We use three different proteins, annexin, streptavidine, and isolectin, to demonstrate high selectivity and high sensitivity biosensor. The biosensor can distinguish at least $17 \mathrm{kDa}$ in molecular weight with $100 \%$ selectivity.

We also examined the effects of surface properties on protein adsorption / displacement using well-defined SAMs of alkane-thiols. Strong interaction of protein with hydrophobic surface induces conformational spreading and transitions to an irreversibly adsorbed state. The strong binding causes the protein displacement very difficult. On the other hand, $\mathrm{COOH}$ - and $\mathrm{OH}-\mathrm{SAM}$ modified hydrophilic surfaces allow very weak binding with proteins, resulting high chance of displacement, thus high selectivity.

\section{REFERENCES}

[1] B.B. Haab, “Antibody Arrays in Cancer Research”, Molecular \& Cellular Proteomics, 4, 4 (2003), pp.377-383

[2] X. Yu, D. Xu, and Q. Cheng, "Label-free detection methods for protein microarrays”, Proteomics, 6 (2006), pp.5494-5503

[3] K.V. Gobi, K. Matsumoto, K.Toko, H.Ikezaki, and N.Miura "Enhanced sensitivity of SAM-based SPR immunosensor for detection of benzaldehyde using a single-step multi-sandwich immunoassay”, Anal. Bioanal. Chem., 387 (2007), pp.2727-2735

[4] R. Chaerkady and A. Pandey "Applications of Proteomics to Lab Diagnosis”, Annu. Rev. Pathol. Mech. Dis.,3 (2008), pp.485-489

[5] T.A. Horbett and J.L.Brash "Proteins at Interfaces II: Fundamentals and Applications”, American Chemical Society, Washington, DC 1995. pp.112-128

[6] C.F.Wertz et al. "Effect of surface hydrophobicity on adsorption and relaxation kinetics of albumin and fibrinogen: single-species and competitive behavior”, Langmuir, 17 (2001), pp.3006-3016

[7] R. A. Latour, Jr. "Biomaterials : Protein-Surface Interactions" Encyclopedia of Biomaterials and Biomedical Engineering (2005), pp.1-15

[8] J.J. Gray, "The interaction of proteins with solid surfaces", Current Opinion in Structural Biology, 14 (2004), pp.110-115 